\title{
Development of a Free Space, LED Illuminated Spectral-domain Optical Coherence Tomography Setup
}

\author{
Nyasha J. Suliali ${ }^{1, *}$, Peter Baricholo ${ }^{1}$, Pieter H. Neethling ${ }^{2}$, Erich G. Rohwer ${ }^{2}$ \\ ${ }^{1}$ Optics Research Group, Department of Applied Physics, National University of Science and Technology, Zimbabwe \\ ${ }^{2}$ Laser Research Institute, Department of Physics, Stellenbosch University, South Africa \\ *Corresponding Author: Nyasha.Suliali@nust.ac.zw
}

Copyright $\bigcirc 2017$ by authors, all rights reserved. Authors agree that this article remains permanently open access under the terms of the Creative Commons Attribution License 4.0 International License

\begin{abstract}
Free-space spectral domain optical coherence tomography has been demonstrated using an $8 \mathrm{~mW}$ ultra-bright $850 \mathrm{~nm}$ light-emitting diode with a $40 \mathrm{~nm}$ spectral width. The system detects longitudinal reflectivity of surface and sub-surface layers of optical elements to depths of a millimetre with high fidelity. Development stages included mathematical analysis of light interference by superposition of electric field phasors of reference and sample arms of a Michelson interferometer. A method by which depth-resolved reflectivity is acquired is described. A locally assembled Czerny Turner monochromator was aligned such that the interferometer output beam is dispersed into its spectral components before image re-construction. Calibration of the 2048-pixel detecting charge-coupled device line camera was performed using a Mercury vapour lamp with 8 spectral lines spanning from the ultra-violet to yellow region of the electromagnetic spectrum. Processing of interference fringe signals from spectral domain data is described and an analysis of variations in frequency of the interference fringe signal and threshold illumination with depth into the sample presented. A test of sensitivity of the depth imaging algorithm to low-amplitude signals is also reported.
\end{abstract}

Keywords Optical Coherence Tomography, Spectral Interferometer, Interferometry, Axial Scan

\section{Introduction}

Optical coherence tomography (OCT) is a leading modality among present-day methods of imaging using electromagnetic energy. In its infancy, ophthalmic imaging was the primary application of OCT systems due to acute response of the eye to light energy. However, research work spread into the field of non-destructive inspection where probing of non-biological samples has been reported for instance, characterisation of the sub-surface morphology of Chinese glazes [1] and defect detection in ceramic dentures [2]. Instantaneous acquisition of multiple reflectors at resolutions of a few microns has singled out Frequency Domain OCT (FD-OCT) from time domain OCT (TD-OCT) and imaging modalities such as Magnetic Resonance Imaging, Computed Tomography, Ultrasound and confocal microscopy [3]. Key properties that attracted researchers to this imaging technique include the non-invasive, non-ionising and non-contact nature of light when interacting with a sample. While OCT has been implemented using optical fibres [4,5], free space implementations still find use especially in educational applications. We developed a free-space SD-OCT setup illuminated by an ultra-bright infra-red light-emitting diode (LED) with a Gaussian spectrum centred at $850 \mathrm{~nm}$. Its spectrum has a 40 $\mathrm{nm}$ FWHM, total optical power of $8 \mathrm{~mW}$ at $20 \mathrm{~mA}$ with a half viewing angle of $55^{\circ}$. A locally assembled power supply unit was used to bias the LED for optimum illumination. This paper outlines the structure of the interferometer and method by which spectra were processed to obtain the axial depth reflectivity profile.

\section{Theory and Methodology}

Development of the OCT system entailed construction of a Michelson interferometer which was set to direct its output beam to a locally assembled Czerny-Turner mount monochromator. The interferometer source was mounted, a beam splitter $B S$ located at origin $O$ and reflectors positioned in the reference and sample arms as illustrated in Figure 1:

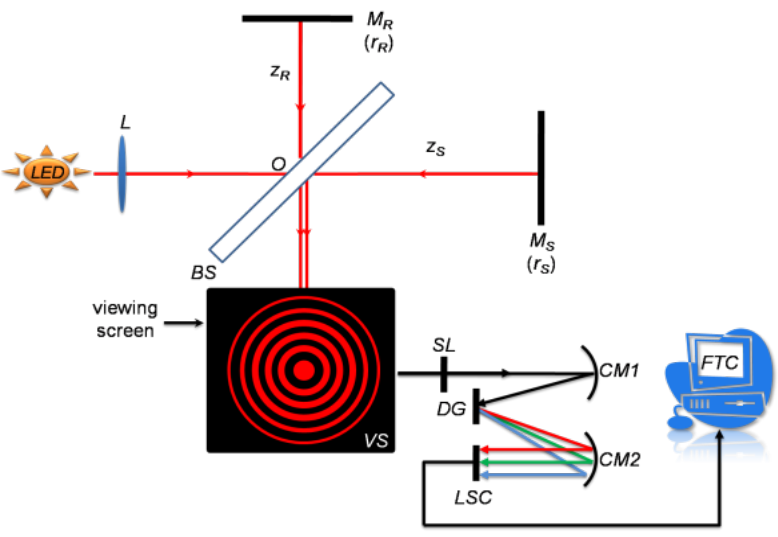

Figure 1. The LED-illuminated SD-OCT setup. 
In this setup, light generated by the LED is partially reflected to reference reflector $M_{R}$ of electric field reflectance $r_{R}$, while the transmitted beam propagates a distance $z_{\mathrm{S}}$ to depth varying reflector $M_{S}$ with reflectance $r_{S}$. On re-combination at $B S$, superposition of the beams generates an output electric field $E(k)$ which is a sum of reference and sample arm electric fields $E_{R}(k)$ and $E_{S}(k)$.

This output beam has an intensity pattern illustrated by the fringes in Figure 1 as viewed on a screen $V S$. When using a quasi-monochromatic light source, interference fringes have a constant visibility or modulation determined by values of minimum and maximum intensity $I_{\min }$ and $I_{\text {max }}$ given by [6]:

$$
V=\left(I_{\max }-I_{\min }\right) /\left(I_{\max }+I_{\min }\right)
$$

For the setup shown in Figure 1, intensity reaches its maximum value when phase difference $\delta=0, m_{e} \pi$ where $m_{e}$ is even. This translates to path differences $\Delta z$ that are an integral number of half wavelengths. Conversely minima are obtained at $\Delta z=n \lambda / 4$ where $n$ is an integer. Path difference therefore dictates the phase of the output beam. With quasi-monochromatic sources, the fringes are therefore visible through large path differences. However, where a broadband source illuminates the interferometer, fringes tend to wash out beyond the zero path difference (ZPD) point or the coherence gate of the light source $[7,8]$. This is the key feature that separates OCT sources from line sources such as Mercury lamps. On adding fields $E_{\mathrm{R}}(k)$ and $E_{\mathrm{S}}(k)$ and multiplying $E(k)$ with its complex conjugate, it can be shown that the spectral interferogram $I(k)$ oscillates in $k$-space according to the analytic solution:

$$
\begin{aligned}
I(k) & =r_{R}^{2} \xi^{2} S(k)+\xi^{2} S(k)\left[\int_{\zeta_{1}}^{\zeta_{2}} r\left(z_{S}\right) e^{-j k\left(2 z_{S}\right)} d z_{S}\right]^{2} \\
& +2 \xi^{2} S(k) r_{R} \int_{\zeta_{1}}^{\zeta_{2}} r\left(z_{S}\right) e^{-j k\left(2 z_{S}\right)} d z_{S}
\end{aligned}
$$

where amplitude reduction factor $\xi$ represents a product of the reflectivity and transmissivity of $B S$ as the reference and sample arm beam pass through these elements.
Reference and sample arm self intereference is shown by squared terms while the interference signal is an integral of the product of two functions: the reflectivity of the sample reflector located at depth position i.e. $r\left(z_{S}\right)$ and a phase shift term $e^{-j k\left(2 z_{S}\right)}$. The integrated product also describes the capability of obtaining multiple reflections from a single sample that has sub-surface layers at varying depths. Now, the objective of low coherence interferometry is to extract this depth-resolved data $[9,10]$ which in this case is achieved by an inverse Fourier Transform. The depth resolved data defines the axial scan of the OCT system.

To re-construct a single slice i.e. a cross-sectional image, axial scans of several transversally separated spots can be obtained by scanning the sample at adjacent points. Similarly, a three dimensional image can be re-constructed by performing slice scans throughout the height of a sample. Image pixel size can then be defined by the spot size of the beam at every scanned point. The system was therefore illuminated at $850 \mathrm{~nm}$ with a $40 \mathrm{~nm}$ line width. Alignment was carried out using a $1 \mathrm{~mW}, 633 \mathrm{~nm}$ Helium Neon gas laser. Interferogram acquisition was achieved by constructing a monochromator made of vertical slit $S L$ that created a vertical image of the output beam directed to concave mirror $C M 1$. Off $C M 1$, the beam propagates to a blazed diffraction grating $D G$ which disperses it into spectral components. The first order diffracted pattern was selected by rotating the grating to direct it to mirror CM2 which focused it onto a linear array of 2048 CMOS detectors of line-scan camera $L S C$. Therefrom, the line-scan camera program was run to open and close its shutter, thereby acquiring the beam spectrum.

Calibration of the spectrometer was carried out using a Mercury vapour lamp which has 8 lines of wavelengths quantified by Monk [11]. The wavelengths were first identified by colour and each line attached to the pixel location. A linear function of wavelength against pixel number resulted in a calibration function used to generate the wavelength data to plot the spectral lines against. Figure 2 shows distribution of the wavelengths of the calibration lamp:

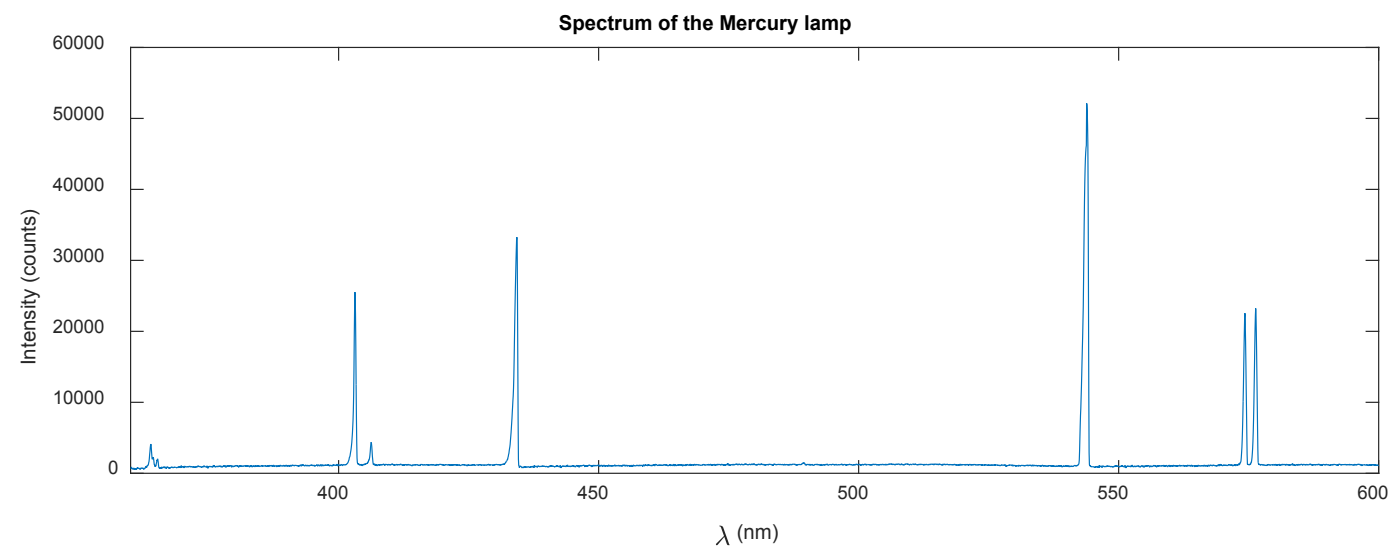

Figure 2. Spectrum of the Mercury lamp 
The strong green line dominates emission at $540 \mathrm{~nm}$, followed by the blue at $435.8 \mathrm{~nm}$, the first violet line at $404.7 \mathrm{~nm}$, the yellow doublet at $577 \mathrm{~nm}$ and $579.1 \mathrm{~nm}$. Sensitivity of the spectrometer was $0.06 \mathrm{~nm} / \mathrm{pixel}$, sufficient a resolution to distinguish between yellow doublet lines. Since measured data would then be shown in the wavelength regime, measured spectra were re-sampled by interpolation in order to achieve even sampling of spectra in $k$-space. An interpolation function was therefore inserted into the fringe processing algorithm to pre-condition all spectra for Fourier transformation. The spectrometer calibration function was therefore key in interpreting fringe separation of the broadband LED. Results of spectra measured and the axial scans computed are presented in section 3 .

\section{Results}

Spectrometer measurements used to determine sensitivity are shown in Table 1. These were performed in the region containing two UV(A), two violets and a strong blue line.

Table 1. Mercury lamp wavelengths identified at five pixel positions.

\begin{tabular}{|c|c|c|}
\hline Pixel Number & Line & $\boldsymbol{\lambda}(\mathbf{n m})$ \\
\hline 67 & UV(A)_1 & 365.0 \\
\hline 89 & UV(A)_2 & 366.4 \\
\hline 732 & Violet_1 & 404.7 \\
\hline 785 & Violet_2 & 407.8 \\
\hline 1260 & Blue & 435.8 \\
\hline
\end{tabular}

The sensitivity parameter obtained from this experiment was $0.06 \mathrm{~nm} /$ pixel. Using this spectrometer, the four key spectra required to compute the axial scan i.e. the source, reference, sample and output arm spectrum were obtained as shown by measurements in Figure 3. The plot shows normalised intensity of the output beam plotted as a function of wavelength for ease of identifying source characteristics. The source spectrum $S(k)$ is represented by $S(\lambda)$, reference spectrum $I_{R}(k)$ by $I_{R}(\lambda)$, sample arm spectrum $I_{S}(k)$ by $I_{S}(\lambda)$ and the intereference fringe signal $I(k)$ by $I(\lambda)$.

Use of a 50/50 beam splitter is confirmed by amplitudes of $I_{R}(\lambda)$ and $I_{S}(\lambda)$ which range at half the magnitude of $S(\lambda)$. However, reflectance $r_{R}$ was greater than $r_{S}$ hence the relationship $I_{R}\left(\lambda_{0}\right)>I_{S}\left(\lambda_{0}\right)$ observable in Figure 3 .

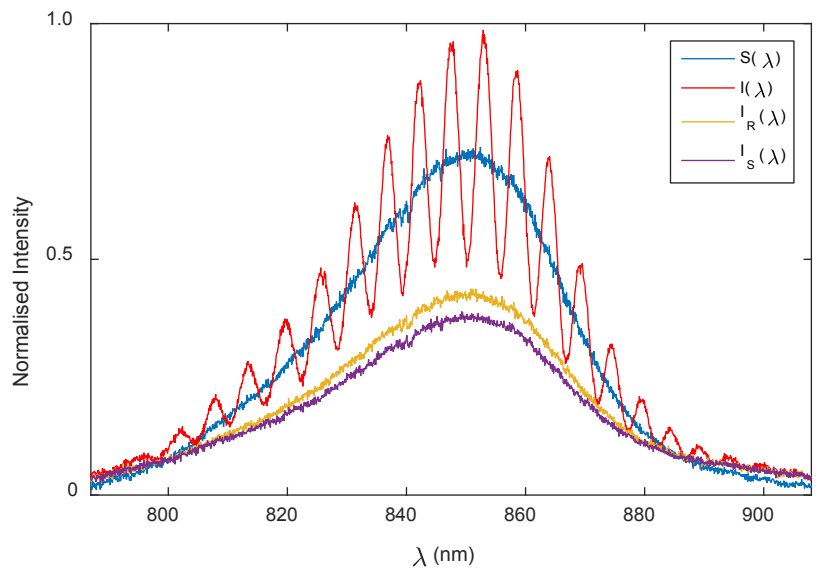

Figure 3. Spectra required to compute $\boldsymbol{r}\left(\mathbf{z}_{S}\right)$.

Modulation of the fringe signal $I(\lambda)$ on $S(\lambda)$ is readily noticeable across the entire spectrum, necessitating de-modulation before performing Fourier transformation. The de-modulated signal is shown to have spectral oscillations in Figure 4.

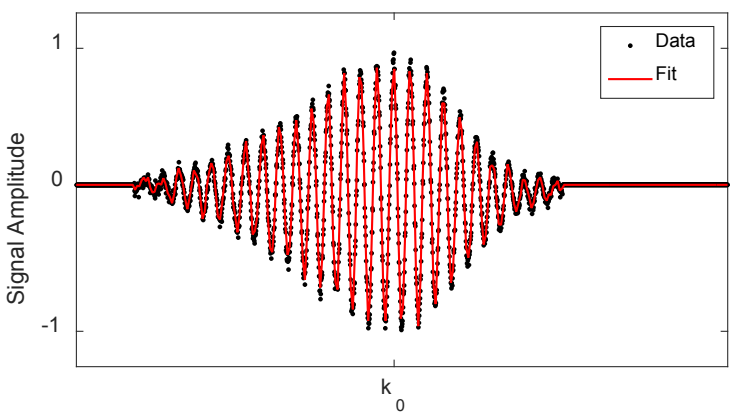

Figure 4. The zero-padded de-modulated fringe signal

Figure 4 shows spectral oscillations centred on centre wave number $k_{0}$ that corresponds to centre wavelength $\lambda_{0}$. Oscillations of the signal represent interference fringes that were extracted off the modulation function of the source. Having obtained spectral distribution, the next task was to convert this signal from the spatial frequency $(k)$ to space domain $z$. To verify validity of the function that computed $\boldsymbol{r}\left(\boldsymbol{z}_{S}\right)$, a correlation experiment was carried out. In this experiment, the sample arm reflector $M_{S}$ was moved away from $O$ from the ZPD point along the axis of the beam. Results in Figure 5 show structure of the interference signal $I(k)$ : 
I(k)

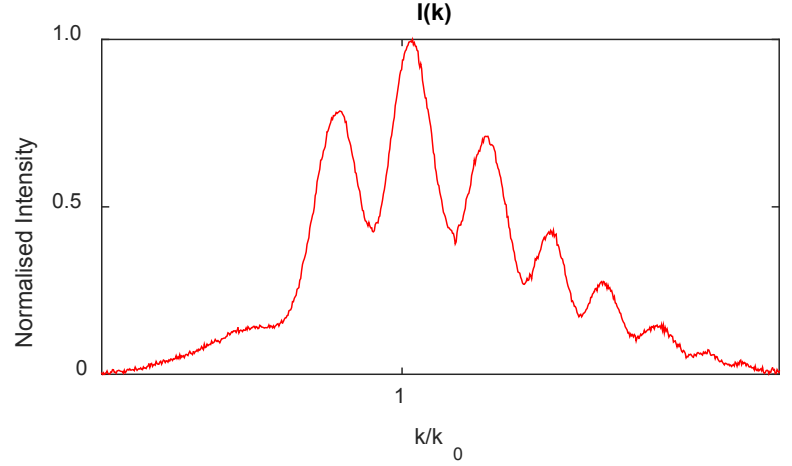

(a)

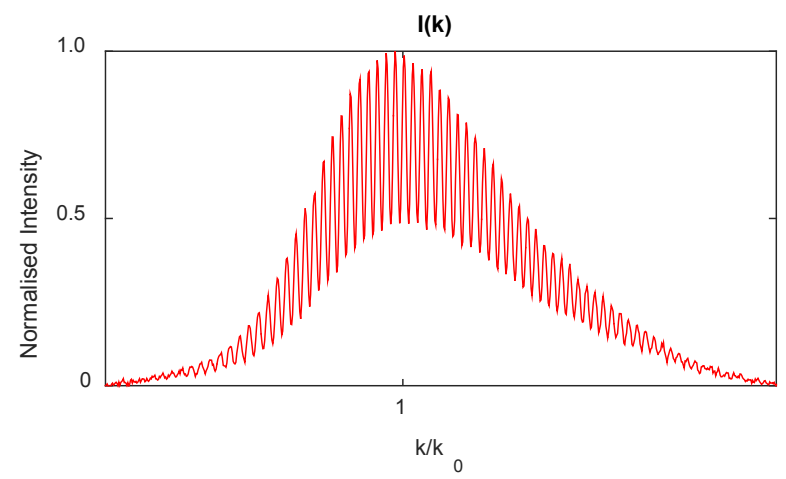

(c)

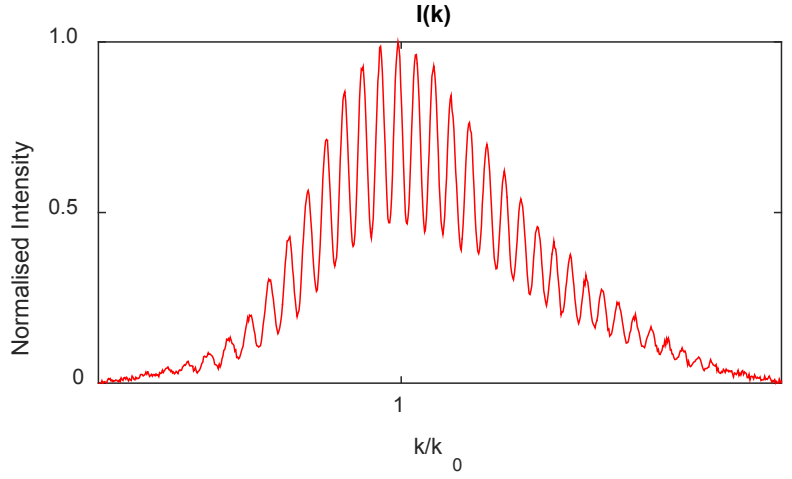

(b)

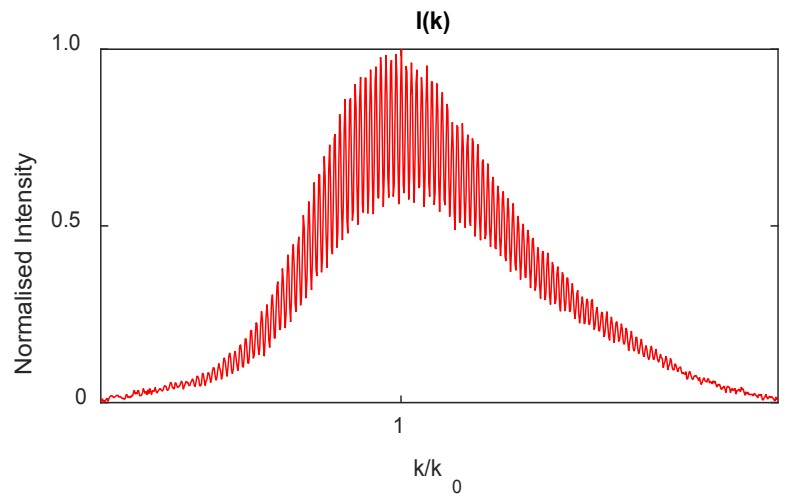

(d)

Figure 5. Plot of $I(k)$ as $z_{\mathrm{S}}$ was increased from the ZPD point by (a) $50 \mu \mathrm{m}$, (b) $110 \mu \mathrm{m}$, (c) $210 \mu \mathrm{m}$ and (d) $380 \mu \mathrm{m}$.

As the beam traversed deeper from the ZPD point to reach the sample, the frequency of interference fringes increased. This observation concurs with theory that dictates congestion of the field of view with fringes as path difference increases [12]. While the contrast of fringes resulted in a high fidelity fringe signal (shown by large fringe amplitude), the threshold illumination or d.c. value of the central spectral component rose with axial depth. Table 2 lists distances through which the reflector was translated range from $50 \mu \mathrm{m}$ to $860 \mu \mathrm{m}$. It is of interest to note that a setting of the reflector within the coherence gate of the source rather than at the exact ZPD produced the fringes as previously observed $[3,4,5,7]$. This quasi-aberrant measurement is often exhibited by readings of $Z_{S A}$ near ZPD e.g. $Z_{S 1}$ and $Z_{S 2}$ which significantly deviate from $Z_{S}$ thereby confirming that geometrical ZPD set when an experimenter observes maximum fringe contrast hardly matches the ideal optical ZPD.
Table 2. Computed depths $\left(z_{S A}\right)$ recorded as $z_{S}$ was increased from 50 $\mu \mathrm{m}$ to $860 \mu \mathrm{m}$.

\begin{tabular}{|c|c|c|c|c|}
\hline $\begin{array}{c}\text { Reflector } \\
\text { Position }\end{array}$ & $\begin{array}{c}(L \pm 0.005) \\
\mathrm{mm}\end{array}$ & $\begin{array}{c}(\Delta L \pm 5) \\
\mu \mathrm{m}\end{array}$ & $\begin{array}{c}\left(z_{S} \pm 5\right) \\
\mu \mathrm{m}\end{array}$ & $\begin{array}{c}\left(z_{S A} \pm 5\right) \\
\mu \mathrm{m}\end{array}$ \\
\hline$z_{S 1}$ & 83.89 & 50 & 50 & 26 \\
\hline$z_{S 2}$ & 83.87 & 70 & 70 & 56 \\
\hline$z_{S 3}$ & 83.83 & 110 & 110 & 113 \\
\hline$z_{S 4}$ & 83.79 & 150 & 150 & 157 \\
\hline$z_{S 5}$ & 83.73 & 210 & 210 & 219 \\
\hline$z_{S 6}$ & 83.65 & 290 & 290 & 297 \\
\hline$z_{S 7}$ & 83.56 & 380 & 380 & 387 \\
\hline$z_{S 8}$ & 83.48 & 460 & 460 & 471 \\
\hline$z_{S 9}$ & 83.40 & 540 & 540 & 551 \\
\hline$z_{S 10}$ & 83.24 & 700 & 700 & 710 \\
\hline$z_{S 11}$ & 83.08 & 860 & 860 & 870 \\
\hline
\end{tabular}


To correlate translation stage distance with distance computed by the algorithm, data of the latter were plotted against the earlier to obtain the graph in Figure 6:

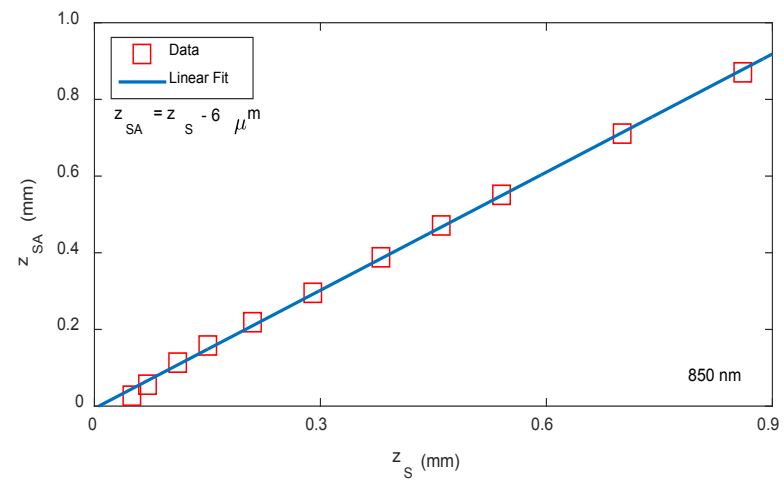

Figure 6. Comparison of computed against translation stage axial depth.
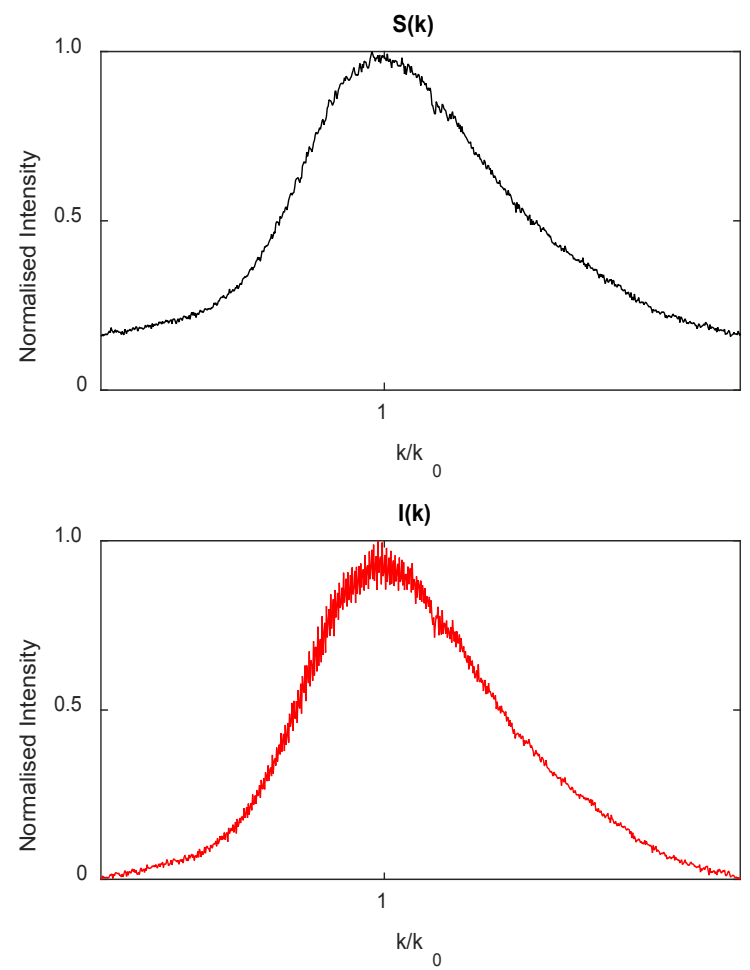

The graph shows unity correlation of algorithm computations of the axial scan from measured spectra. The intercept of $-6 \mu \mathrm{m}$ indicates mismatch between translation stage and optical depths at the ZPD point. This result concurs with depth scan calibration work reported by Choma and co-workers [4] as they tested possibility of variation in coherence length of a swept laser source. To consolidate the analysis of OCT axial imaging presented in the earlier results, the reflector was traversed to a depth near the wash-out point of the interferometer. This experiment tested sensitivity of the depth computation algorithm to signals of the lowest possible magnitude. Figure 7 shows a plot of all four spectra obtained at the maximum possible depth of the interferometer:
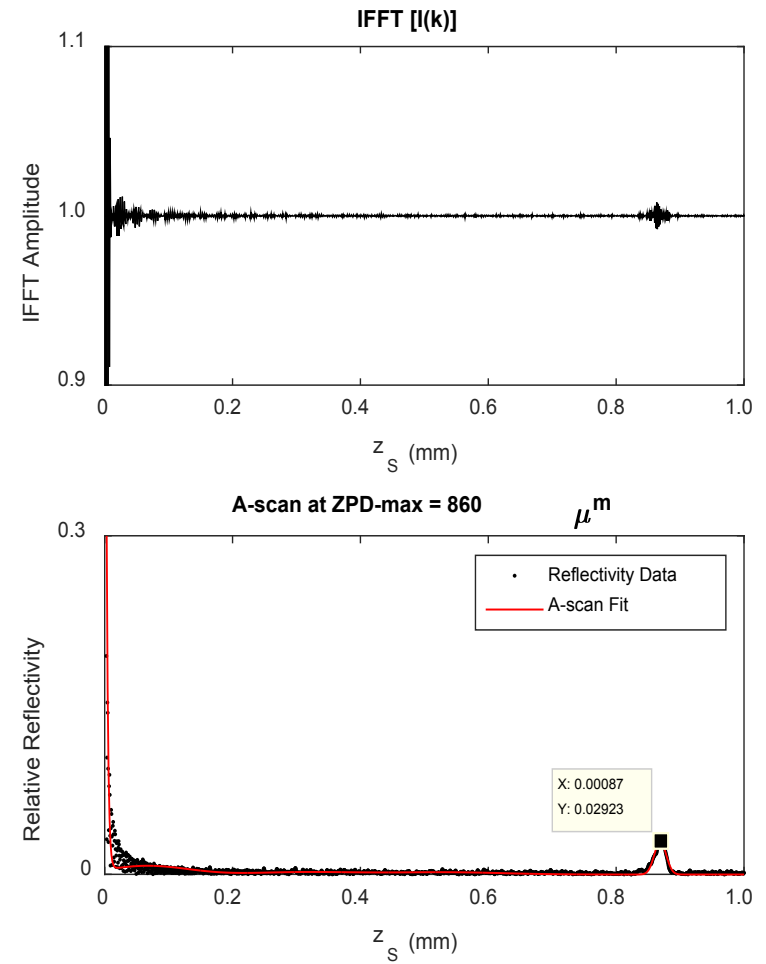

Figure 7. $I(k)$ and axial scan at maximum depth. 
The source spectrum $S(k)$ is shown to be distributed with the wide FWHM of $40 \mathrm{~nm}$. Output spectrum $I(k)$ has such high a frequency of oscillation that the fringes are not observable on the spectrometer screen. Evidence of spectral proximity to the wash out point is in the signal to d.c. ratio which by inspection and calculation, is less than $50 \%$. The algorithm was therefore tested to an extreme case of a low-amplitude interference signal. It is interesting to find that the algorithm developed in this work was capable of detecting the axial depth at which this reflector was located. The axial scan graph in Figure 7 then shows that axial depth was $870 \mu \mathrm{m}$. This result confirms the necessity of removing the modulating source spectrum from $I(k)$ such that depth resolved information can still be detected at the furthest reflector positions.

In terms of applications, the developed OCT system can be used for absolute displacement measurement in the micrometer-regime, surface profilometry and to detect sub-surface reflections to depths of a millimetre. Refractive index of reflective materials a millimetre thick, can also be carried out with the developed system.

\section{Conclusions}

A free space SD-OCT system was developed and implemented in absolute axial displacement measurement. Imaging depths of up to a millimetre were demonstrated as reported in Figures 6 and 7. The frequency content of the LED showed wide distribution $(40 \mathrm{~nm})$ of wavelengths about the mean of $850 \mathrm{~nm}$. Beside increase in fringe frequency, self-interference contributed d.c. values that dominated interferogram data at axial positions near the wash-out point. Low signal to d.c. ratio was countered by de-modulation of the fringe signal from the output spectrum. With such sensitivity, this system could be applicable in imaging of biological samples that tend to scatter light shone on them. The surface structure of optical elements can equally be re-constructed using this system.

\section{Acknowledgements}

We acknowledge the African Laser Center and Laser Research Institute (South Africa), Research Board of the National University of Science and Technology (Zimbabwe) and The World Academy of Sciences for funding this work.

\section{REFERENCES}

[1] Yang ML, Winkler AM, Barton JK, Vandiver PB. Using optical coherence tomography to examine the subsurface morphology of Chinese glazes. Archaeometry. 51(5):808 - 821, September 2008.

[2] Sinescu C, Bradu A, Topala FI, Negrutiu ML, Duma VF, Podoleanu AG. Integral ceramic superstructure evaluation using time domain optical coherence tomography. In: Lasers in Dentistry XX; San Fransisco. p. 89290N-1 - 89290N-7, 2014.

[3] Fujimoto JG, Chen Y, Aguirre A. Biomedical Imaging using Optical Coherence Tomography. In: Fujimoto JG, Farkas DL, editors. Biomedical Optical Imaging. New York: Oxford University Press, 2009.

[4] Choma MA, Hsu K, Izatt JA. Swept source optical coherence tomography using an all-fiber 1300-nm ring laser source. Journal of Biomedical Optics. 10(4):044009-1 - 6, August 2005.

[5] Zhang K, Kang JU. Principles of Optical Coherence Tomography. In: Kang JU, editor. Fiber Optic Sensing and Imaging. London: Springer Science and Business Media, 2013.

[6] Born M, Wolf E. Principles of Optics. 7th ed. London: Cambridge University Press, 1999.

[7] Jansz P, Richardson S, Wild G, Hinckley S. Modeling of low coherence interferometry using broadband multi-Gaussian light sources. Photonic Sensors. 2(3):247 - 258, 2012.

[8] Kramoreva LI, Rozhko YI. Optical coherence tomography. Journal of Applied Spectroscopy. 77(4):449 - 467, August 2010 .

[9] Brezinski ME, Tearney GJ, Weissman NJ, Boppart SA, Bouma BE, Hee MR, Weyman AE, Swanson EA, Southern JF, Fujimoto JG. Assessing atherosclerotic plaque morphology: comparison of optical coherence tomography and high frequency intravascular ultrasound. Heart. 77:397 - 403, 1997.

[10] Zvyagin AV, Garcia-Webb MG, Sampson DD. Semiconductor line source for low-coherence interferometry. Applied Optics. 40(6):913 - 915, February 2001.

[11] Monk GS. Light: Principles and Experiments. 1st ed. Chicago: McGraw-Hill Book Company, 1937.

[12] Izatt JA, Choma MA. Theory of Optical Coherence Tomography. In: Drexler W, Fujimoto JG, editors. Optical Coherence Tomography: Technology and Applications. Springer Science and Business Media. p. 47 - 72, 2008. 